\title{
Effect of acetabular morphological parameters applied in proximal femoral varus osteotomy on the treatment of developmental dysplasia of the hip in children
}

\author{
Shenghua Qiu, Haiwei Lin, Meng Xu, Chengliang Liu, Haifeng Wang, Qingwei Cao, Jinxiang Liu \\ Department of Pediatric Surgery, Linyi Central Hospital, Linyi, China \\ Contributions: (I) Conception and design: S Qiu; (II) Administrative support: J Liu; (III) Provision of study materials or patients: S Qiu, J Liu; (IV) \\ Collection and assembly of data: S Qiu, H Lin; (V) Data analysis and interpretation: J Liu; (VI) Manuscript writing: All authors; (VII) Final approval \\ of manuscript: All authors. \\ Correspondence to: Jinxiang Liu. Department of Pediatric Surgery, Linyi Central Hospital, 17 Jiankang Road, Yishui, Linyi 276400, China. \\ Email: fug5973@163.com.
}

\begin{abstract}
Background: The study was carried out based on the background that though proximal femoral varus osteotomy is one of the most effective methods in treating developmental dysplasia of the hip (DDH), its surgical efficiency and effect need to be improved.

Methods: The neck shaft angle and acetabular morphological parameters were measured before and after operation and during clinical follow-up. The Severin criteria and McKay criteria were both adopted to score the measurements. The distance from the center of the femoral head to the medial cutting bone (expressed as $\mathrm{C}$ value) and the shortened lengths of the proximal femur (expressed as $\Delta \mathrm{S}$ ) calculated by formula and obtained by actual measurement were compared.

Results: The neck shaft angle and acetabular morphological parameters after operation and during clinical follow-up were significantly lower than those before operation $(\mathrm{P}<0.05)$. The proportion of children with McKay scores of I, II, III, and IV after operation was 50.00\%, 41.67\%, 8.33\%, and 0.00\%, respectively; while the proportion of children with Severin scores of I, II, III, and IV was $55.00 \%, 38.33 \%, 6.67 \%$, and $0.00 \%$, respectively. There were no significant differences in the $\mathrm{C}$ value and $\Delta \mathrm{S}$ obtained after formula calculation and actual measurement $(\mathrm{P}>0.05)$.
\end{abstract}

Conclusions: The acetabular morphological parameters applied in proximal femoral varus osteotomy are of great value for the treatment of DDH in children.

Keywords: Proximal femoral varus osteotomy; developmental dysplasia of the hip (DDH); neck shaft angle; acetabular index; parameters

Submitted Apr 02, 2021. Accepted for publication May 18, 2021.

doi: $10.21037 / \mathrm{tp}-21-200$

View this article at: http://dx.doi.org/10.21037/tp-21-200

\section{Introduction}

Developmental dysplasia of the hip (DDH) is manifested by developmental dysplasia of the acetabulum and developmental malformation of the lower limbs in children with dislocated hip joints (1-3). The study has indicated that the Formation of DDH is related with environmental and genetic factors which could directly affect the developments of the connective tissue, osteogenesis, and chondrogenesis (4).
Although the incidence of DDH is not currently very high, it has a serious impact on the daily activities and psychological development of children $(5,6)$. Therefore, it is of positive significance to adopt effective treatment for DDH. Proximal femoral varus osteotomy, as one of the most effective methods for the treatment of DDH, has been the subject of clinical testing for decades and has been supported by many clinical researchers (7-9). However, 
Table 1 General information of participants

\begin{tabular}{lc}
\hline General information & Number $(\mathrm{n}=60)$ \\
\hline Age (years) & $2-10$ \\
Average age (years) & $5.36 \pm 3.49$ \\
Male-female ratio & $1: 2$ \\
Height $(\mathrm{cm})$ & $103 \pm 9.93$ \\
Weight $(\mathrm{kg})$ & $18.6 \pm 1.37$ \\
Left side of hip bone (cases) & 32 \\
Right side of hip bone (cases) & 28 \\
\hline
\end{tabular}

its effect is often related to a variety of factors, including age, development of the acetabulum, and dislocation of the hip joint. Before operation, physicians often measure patients' neck shaft angle to obtain accurate parameters, thus improving the operation efficiency $(10,11)$. In addition, femur length after osteotomy is one of the important indicators that should be clarified intraoperatively, as its accuracy is the main factor affecting the operation effect $(12,13)$. Based on this, this study aimed to analyze the changes of neck shaft angle, acetabular index, $\mathrm{C}$ value, and $\Delta \mathrm{S}$ during proximal femoral varus osteotomy, and evaluate them in combination with Severin and McKay scores after operation, with the purpose of providing references for the obvious therapeutic effect of proximal femoral varus osteotomy on DDH. We present the following article in accordance with the STROBE reporting checklist (available at http://dx.doi.org/10.21037/tp-21-200).

\section{Methods}

\section{General information}

All procedures performed in this study involving human participants were in accordance with the Declaration of Helsinki (as revised in 2013) (14). This study was approved by the Linyi Central Hospital Ethics Committee (20180312). A total of 60 children with DDH admitted to our hospital from May 2018 to May 2020 were selected as the study participants and their general information is shown in Table 1.

\section{Inclusion and exclusion criteria}

The inclusion criteria were as follows: (I) the guardians of the participants were informed of the detailed contents of this study; (II) the guardians of the participants voluntarily provided written informed consent; (III) child patients with DDH aged 2-10 years old.

The exclusion criteria were as follows: (I) hip dislocation in children that was caused by trauma or pathological reasons; (II) the guardians of the children refused the postoperative follow-up.

\section{Study methods}

\section{Preoperative preparation}

All participants' hips were photographed and reconstructed by three-dimensional computed tomography (3D-CT) with $\mathrm{X}$-ray before operation, and the neck shaft angle and acetabular index were recorded. Then, the operative implementation plan was formulated according to these parameters.

\section{Operation methods}

All participants were treated with proximal femoral varus osteotomy, and appropriate anesthetics were injected according to their individual conditions. During the operation, the participants took supine positions, and their affected side was increased by $30^{\circ}$. During the operation, the skin, subcutaneous tissues, and deep fascia on the affected side of were incised, and rectus femoris was exposed by separating the sartorius muscles and tensor fasciae latae. Then, the epiphysis of the hip was incised, lateral epiphysis and periosteum were stripped, and the gluteus medius and gluteus minimus were stripped after hemostasis. The lateral circumflex femoral artery was separated, ligated, and cut. Next, the fat was pushed away to fully expose the articular capsule which was then cut in a T-shape, the insertion point of the round ligament was cut off, and the femur was externally rotated to expose the acetabulum. The hyperplastic tissues were then removed, and the acetabular labrum was incised. The iliopsoas was isolated, cut off from the outer edge, and clamped and marked by Kocher's forceps. The flap was cut backwards along the middle of the incision, and the fascia lata was cut longitudinally at the greater trochanter to expose the patients' upper femur. Subsequently, the periosteum was separated, and an appropriately sized steel plate was selected for pre-bending in advance. At the same time, the proximal femur was fixed with 2 screws, 2 Kirschner wires were vertically implanted into the proximal and distal ends of the osteotomy, and the anteversion angle of the participant was measured. The femur was amputated and rotated so that its anteversion 
angle ranged from $10^{\circ}$ to $25^{\circ}$, with the angle at osteotomy greater than $120^{\circ}$ but slightly less than normal value. The shortened lengths of the femoral osteotomy were consistent with the prolapse height of the femoral head. When resetting the osteotomy end, the proximal and distal screws were tightened in turn and the soft tissues were cleaned at the upper edge of the acetabulum. Taking the center of Y-shaped cartilage as the axis, the external iliac plate was severed at $15 \mathrm{~mm}$ from the upper edge of the acetabulum, the distal end of the osteotomy was turned forward, outward, and downward, and the gap was filled with bone blocks. Flexion, internal and external rotation of children's affected limb were carried out, and appropriate stretching of the distal limb was also performed to form a gap of about $1 \mathrm{~cm}$ between the femur and acetabulum. The absence of dislocation of the femur represented the completion of reduction. Then, the joint capsule was sutured, maintaining the hip joints in flexion and abduction of $30^{\circ}$ and internal rotation of $20-30^{\circ}$. Finally, the epiphysis and various layers of tissues were sutured and bandaged, and plaster was used for external fixation of the affected side. After operation, $\mathrm{X}$-rays were taken of the affected side.

\section{Efficacy evaluation}

In this study, the participants undergoing proximal femoral varus osteotomy were followed up 1 year after surgery, and the parameters such as neck shaft angle and acetabular index were statistically analyzed and compared with their preoperative parameters to evaluate the therapeutic effect of the operation.

\section{Evaluation criteria}

The Severin criteria were assessed according to acetabular morphology, and the effect of surgical intervention was divided into grade I, II, III, and IV. The number of participants in each grade was recorded.

The McKay criteria were assessed according to participants' movements, walking patterns, and pains. The effect of surgical intervention was divided into grade I, II, III, and IV, and the number of participants in each grade was recorded.

The shortened lengths of femur were calculated after operation. The midpoint of the lesser trochanter was taken as the horizontal plane of osteotomy, the horizontal plane of the midpoint of the lesser trochanter was set as osteotomy line $\mathrm{b}$, and the medial point of the osteotomy line was $\mathrm{P}_{2}$.
Taking the femoral head as a circle, the center of the circle was $\mathrm{P}_{1}$, the distance between $\mathrm{P}_{1}$ and $\mathrm{P}_{2}$ was defined as $\mathrm{c}$, and the distance between $\mathrm{P}$ - and $\mathrm{b}$ before the osteotomy was $\mathrm{S}_{1}$. The angle between $\mathrm{c}$ and $\mathrm{b}$ before operation was set as $\theta_{1}$, the angle between $\mathrm{c}$ and $\mathrm{b}$ after operation was set as $\theta_{2}$, and the varus angulation was $\theta_{1}-\theta_{2}$. In addition, the distance between $\mathrm{P} 1$ ' and $\mathrm{b}$ after operation was set as $\mathrm{S}_{2}$, and $\Delta \mathrm{S}$ represented the shortened lengths of femur after operation, thus: $\Delta S=c\left(\sin \theta_{1}-\sin \theta_{2}\right)$.

The actual shortened lengths of the femur were measured after operation. The software Digimizer V4.6.1 (https://www.digimizer.com, MedCalc Software, Ostend, Belgium) was adopted to measure the postoperative and preoperative hip $\mathrm{X}$-rays. We set $\mathrm{P} 1$ ' as the intersection point between the midline of the femoral neck and the midpoint of the growth plate of the proximal femur. The line from $\mathrm{P} 1$ ' to the innermost point of the osteotomy line, $\mathrm{P} 2$ ', was c. The line of $\mathrm{P} 1$ ' intersected vertically with the osteotomy line at point $\mathrm{N}$ and the distance between the 2 points was $\mathrm{S}_{2}$. The preoperative $\mathrm{X}$-ray was introduced, and the intersection point $\mathrm{P}_{2}$ of the same line length as $\mathrm{c}$ was made from the measurement site $\mathrm{P} 1$ of the femoral head to the inner cortex of the femur as the osteotomy position, and its perpendicular line with the midline of the femoral shaft was taken as the osteotomy line. The osteotomy line and P1 intersected vertically at point $M$, and the distance between the 2 points before operation could be measured as $S_{1}$, thus: $\Delta \mathrm{S}=\mathrm{S}_{1}-\mathrm{S}_{2}$.

\section{Statistical analysis}

All data were processed using the statistical software SPSS 20.0 (IBM Corp., Chicago, IL, USA). Measurement data were tested by $t$-test and enumeration data were tested by chi-square $\left(\chi^{2}\right)$ test. The differences had statistical significance when $\mathrm{P}<0.05$.

\section{Results}

\section{Comparison of participants' neck shaft angle before and after operation and during clinical follow-up}

The neck shaft angle before and after operation and during clinical follow-up is shown in Figure 1. The participants' neck shaft angle before and after operation and during clinical follow-up was $\left(115.09 \pm 6.92^{\circ}\right),\left(95.09 \pm 5.93^{\circ}\right)$, and $\left(81.91 \pm 4.27^{\circ}\right)$, respectively, and there were significant differences in neck shaft angles before operation and during 


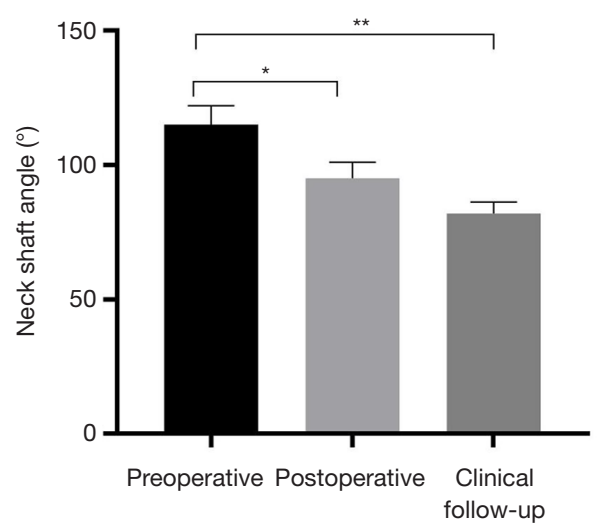

Figure 1 Comparison of neck shaft angle before and after operation and during clinical follow-up. The abscissa axis indicates preoperative, postoperative, and clinical follow-up, while the ordinate axis indicates neck shaft angle. The neck shaft angle before and after operation and during clinical follow-up was $(115.09 \pm 6.92),(95.09 \pm 5.93)$, and $(81.91 \pm 4.27)$, respectively. * indicates that there were significant differences in neck shaft angle before and after operation $(\mathrm{t}=17.00, \mathrm{P}<0.01)$; ** indicates that there were significant differences in neck shaft angle before operation and during clinical follow-up $(\mathrm{t}=31.61, \mathrm{P}<0.01)$.

clinical follow-up $(\mathrm{P}<0.01)$.

\section{Comparison of participants' acetabular index before and after operation and during clinical follow-up}

The acetabular index before and after operation and during clinical follow-up is shown in Figure 2. The acetabular index before and after operation and during the clinical follow-up was $(37.96 \pm 0.49),(18.93 \pm 0.13)$, and $(16.49 \pm 1.03)$, respectively.

\section{McKay score of operation efficacy}

The McKay score of operation efficacy is shown in Table 2. After operation, the number of participants with McKay scores of I, II, II, and IV were 30, 25, 5, and 0, respectively, accounting for $50.00 \%, 41.67 \%, 8.33 \%$, and $0.00 \%$, respectively.

\section{Severin score of operation efficacy}

The Severin score of operation efficacy is shown in Table 3. After operation, the number of participants with Severin scores of I, II, III, and IV, were 33, 23, 4, and 0, respectively,

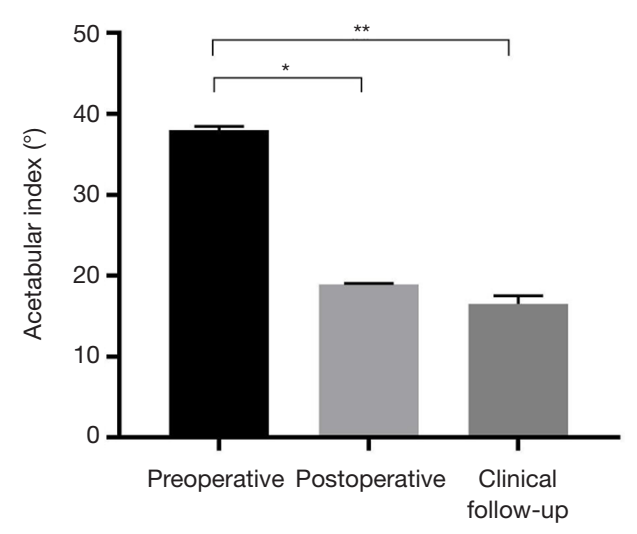

Figure 2 Comparison of participants' acetabular index before and after operation and during clinical follow-up. The abscissa axis indicates preoperative, postoperative, and clinical follow-up, while the ordinate axis indicates acetabular index. The acetabular index before and after operation and during the clinical followup was $(37.96 \pm 0.49),(18.93 \pm 0.13)$, and $(16.49 \pm 1.03)$, respectively. * indicates that there were significant differences in acetabular indexes before and after operation $(\mathrm{t}=290.77, \mathrm{P}<0.01)$; ** indicates that there were significant differences in acetabular indexes before operation and during the clinical follow-up $(\mathrm{t}=145.80, \mathrm{P}<0.01)$.

accounting for $55.00 \%, 38.33 \%, 6.67 \%$, and $0.00 \%$, respectively.

\section{Comparison of actual and calculated distances from the center of the femoral head to the medial proximal end of the osteotomy}

The actual and calculated distances from the center of the femoral head to the medial proximal end of the osteotomy are shown in Figure 3. The actual and calculated distances from the center of the femoral head to the medial proximal end of the osteotomy were $(49.03 \pm 9.01) \mathrm{mm}$ and $(49.01 \pm 8.97) \mathrm{mm}$, respectively, and there were no significant differences between the 2 measurement groups $(\mathrm{t}=0.01$, $\mathrm{P}>0.05$ ).

\section{Comparison of actual and calculated shortened lengths of the proximal femur}

The actual and calculated values of the shortened lengths of the proximal femur are shown in Figure 4. The actual and calculated distances from the center of the femoral head to the medial proximal end of the osteotomy were $(5.97 \pm 3.39) \mathrm{mm}$ and $(5.99 \pm 3.43) \mathrm{mm}$, respectively, and there were no significant 
Table 2 McKay score of operation efficacy

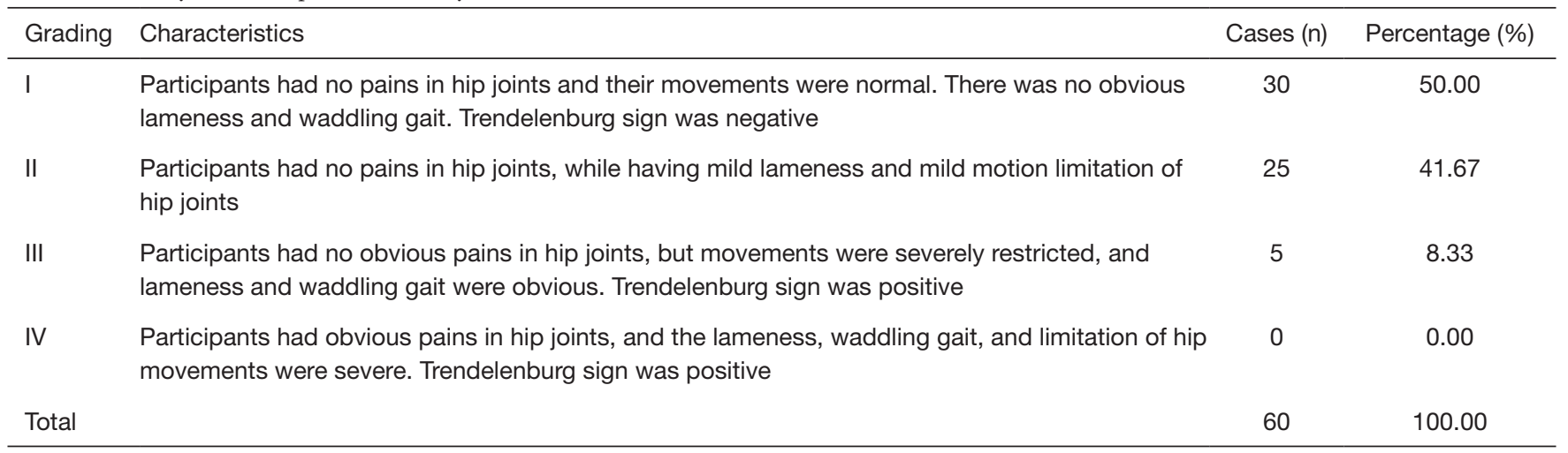

Table 3 Severin score of operation efficacy

\begin{tabular}{|c|c|c|c|}
\hline Grading & Characteristics & Cases (n) & Percentage (\%) \\
\hline II & Certain degree of deformation in the acetabular head & 23 & 38.33 \\
\hline III & Acetabular dysplasia, with continuous Shenton line of the hip & 4 & 6.67 \\
\hline IV & Subluxated hip, with discontinuous Shenton line of the hip & 0 & 0.00 \\
\hline
\end{tabular}

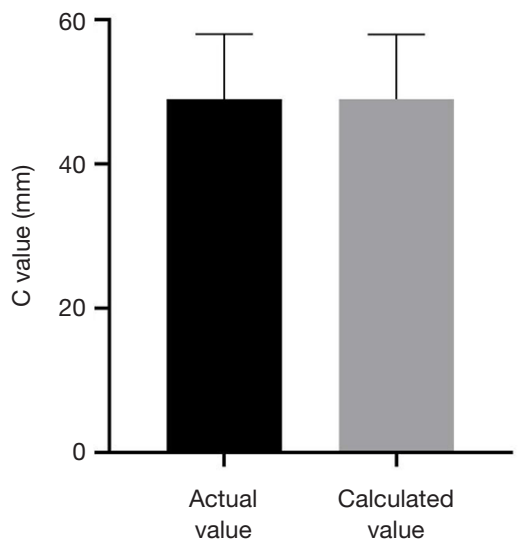

Figure 3 Comparison of actual and calculated distances from the center of the femoral head to the medial proximal end of the osteotomy. The abscissa axis indicates actual value and calculated value, while the ordinate axis indicates $C$ value. The actual and calculated distances from the center of the femoral head to the medial proximal end of the osteotomy were $(49.03 \pm 9.01) \mathrm{mm}$ and $(49.01 \pm 8.97) \mathrm{mm}$, respectively, and there were no significant differences between the 2 calculations $(\mathrm{t}=0.01, \mathrm{P}>0.05)$.

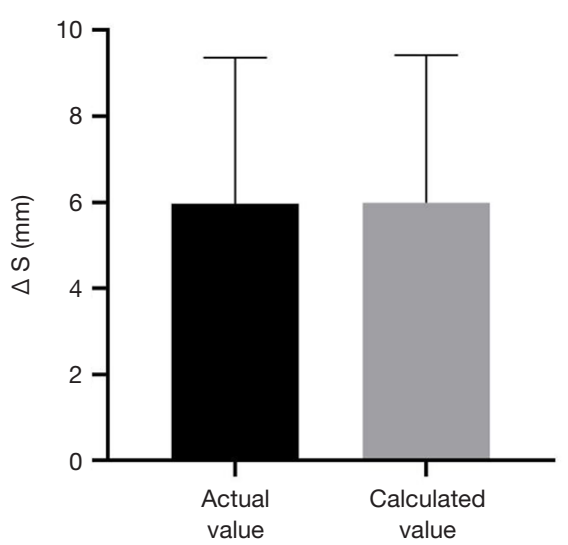

Figure 4 Comparison of actual and calculated shortened lengths of proximal femur. The abscissa axis indicates actual value and calculated value, while the ordinate axis indicates $\Delta \mathrm{S}$. The actual and calculated distances from the center of the femoral head to the medial proximal end of the osteotomy were $(5.97 \pm 3.39) \mathrm{mm}$ and $(5.99 \pm 3.43) \mathrm{mm}$, respectively, and there were no significant differences between the 2 distances $(\mathrm{t}=0.03, \mathrm{P}>0.05)$. 
differences between the 2 methods $(t=0.03, P>0.05)$.

\section{Discussion}

The condition DDH is one of the main causes of developmental malformation of lower limbs in children, and effective clinical intervention is of great significance for promoting healthy development $(15,16)$.

Proximal femoral varus osteotomy is an effective method for treating DDH, but children often experience complications such as increased distance between the femoral head and the inner wall of the acetabulum, dislocation of the femoral head, ischemic necrosis, and so on (17). In children with DDH, chronic compression of the hip bones causes deformation and heterotopia of the femoral head, which leads to a significant enlargement of the femoral neck shaft angle. In children with severe $\mathrm{DDH}$, the neck shaft angle can be increased to more than $150^{\circ}(18,19)$. For children with significantly increased neck shaft angles, proximal femoral varus osteotomy is usually adopted to correct it clinically, to induce concentric reduction of the hip joints and improve the combined state of the acetabulum and femoral head, thus reducing the risk of femoral head dislocation (20,21). In this study, after the participants had undergone corrective surgery, the femoral neck shaft angle and acetabular index decreased to some extent, and children with McKay and Severin grades I and II displayed significantly improved rates of $91.67 \%$ and $93.33 \%$, respectively, indicating that proximal varus osteotomy could effectively improve the femoral neck shaft angle and acetabular index, and significantly reduce the limitation of hip joints in children. To an extent, the femoral neck shaft angle and acetabular index can reflect the actual condition of DDH in children. Sonoda et al. (22) found that the neck shaft angle and anteversion angle of the hip joints in children could directly determine the postoperative joint shape, thus providing an effective reference for preoperative planning. In addition, after the proximal femoral varus osteotomy, the femurs of children will be truncated to some extent. Compared with younger ones, older children have a weaker ability of body regulation, and osteotomy may lead to a shorter femur on the affected side compared to normal side, resulting in lower limb length discrepancy and even uncoordinated movements. Umer et al. have confirmed that the patients younger than 5.6 years old have better clinical outcomes than those of older patients (23). Whether or not to perform femoral osteotomy in children usually depends on the feasibility of femoral head reduction.
Studies have revealed that femoral varus can shorten the femur to some extent, and concentric circle reduction can be achieved only by femoral varus if the required truncation length is short (24). Therefore, accurate calculation of the length change caused by femoral varus and the actual truncation distance before operation can effectively evaluate the necessity of osteotomy, thereby reducing the risk of operation to children to some degree. Besides, an osteotomy that is too large will also increase the tension of the joint soft tissues in children, thus increasing the risk of postoperative dislocation (25). In this study, there were no significant differences in the measured and calculated distances between the central point of the femoral head and the medial point of the proximal end of the osteotomy, and there were no significant differences between the 2 in the measurement of the shortened lengths of the proximal femur, suggesting that accurate assessment of femoral length had important clinical value. Gaffney et al. (26) found that femoral length was significantly correlated with the severity of DDH, which had prompted them to construct a statistical model as a preoperative reference.

The study investigated the effect of acetabular morphological parameters applied in proximal femoral varus osteotomy on the treatment of the children with DDH. However, the study lacks the data of the operative effects on some factors, such as complications and age of children, and more observations on operative outcome are limited small cases and long-term follow-up.

In conclusion, this study demonstrated that acetabular morphological parameters applied in proximal femoral varus osteotomy has high application value in the treatment of DDH in children.

\section{Acknowledgments}

Funding: None.

\section{Footnote}

Reporting Checklist: The authors have completed the STROBE reporting checklist. Available at http://dx.doi. org/10.21037/tp-21-200

Data Sharing Statement: Available at http://dx.doi. org/10.21037/tp-21-200

Conflicts of Interest: All authors have completed the ICMJE uniform disclosure form (available at http://dx.doi. 
org/10.21037/tp-21-200). The authors have no conflicts of interest to declare.

Ethical Statement: The authors are accountable for all aspects of the work in ensuring that questions related to the accuracy or integrity of any part of the work are appropriately investigated and resolved. All procedures performed in this study involving human participants were in accordance with the Declaration of Helsinki (as revised in 2013). This study was approved by the Linyi Central Hospital Ethics Committee (20180312). All participants provided written informed consent.

Open Access Statement: This is an Open Access article distributed in accordance with the Creative Commons Attribution-NonCommercial-NoDerivs 4.0 International License (CC BY-NC-ND 4.0), which permits the noncommercial replication and distribution of the article with the strict proviso that no changes or edits are made and the original work is properly cited (including links to both the formal publication through the relevant DOI and the license). See: https://creativecommons.org/licenses/by-nc-nd/4.0/.

\section{References}

1. Alassaf N. Prediction of the requirement of open reduction for developmental dysplasia of the hip. J Int Med Res 2018;46:54-61.

2. Plischuk M, De Feo ME, Desántolo B. Developmental dysplasia of the hip in female adult individual: Site Tres Cruces I, Salta, Argentina (Superior formative period, 4001000 AD). Int J Paleopathol 2018;20:108-13.

3. Wright S, Cotterell E, Schmidt D. Screening for developmental dysplasia of the hip in a rural health district: An analysis of practice. Aust J Rural Health 2018;26:199-205.

4. Harsanyi S, Zamborsky R, Kokavec M, et al. Genetics of developmental dysplasia of the hip. Eur J Med Genet 2020;63:103990.

5. Talbot C, Adam J, Paton R. Late presentation of developmental dysplasia of the hip: a 15 -year observational study. Bone Joint J 2017;99-B:1250-5.

6. Nie K, Rymaruk S, Paton RW. Clicky hip alone is not a true risk factor for developmental dysplasia of the hip. Bone Joint J 2017;99-B:1533-6.

7. Greber EM, Pelt CE, Gililland JM, et al. Challenges in Total Hip Arthroplasty in the Setting of Developmental Dysplasia of the Hip. J Arthroplasty 2017;32:S38-S44.
8. Dogan O, Caliskan E, Gencer B, et al. Is male gender a prognostic factor for developmental dysplasia of the hip? Mid-long-term results of posteromedial limited surgery. Acta Orthop Traumatol Turc 2019;53:340-5.

9. Kuriyama S, Watanabe $M$, Nakamura $S$, et al. Large medial proximal tibial angles cause excessively medial tibiofemoral contact forces and abnormal knee kinematics following open-wedge high tibial osteotomy. Clin Biomech (Bristol, Avon) 2020;80:105190.

10. Simonsen PO, Hertz JM, Søballe K, et al. Association between periacetabular osteotomy and hip dysplasia among relatives: a cross-sectional study. Hip Int 2019;29:424-9.

11. Yan L, Wang P, Tang C, et al. Effect of acetabular tilt angle on acetabular version in adults with developmental dysplasia of the hip. Zhongguo Xiu Fu Chong Jian Wai Ke Za Zhi 2017;31:647-52.

12. Shi XT, Cheng CM, Feng CY, et al. Crowe Type IV Hip Dysplasia Treated by THA Comebined with Osteotomy to Balance Functional Leg Length Discrepancy: A Prospective Observational Study. Orthop Surg 2020;12:533-42.

13. Shi XT, Li CF, Han Y, et al. Total Hip Arthroplasty for Crowe Type IV Hip Dysplasia: Surgical Techniques and Postoperative Complications. Orthop Surg 2019;11:966-73.

14. World Medical Association. World Medical Association Declaration of Helsinki: ethical principles for medical research involving human subjects. JAMA 2013;310:2191-4.

15. Kural B, Devecioğlu Karapınar E, et al. Risk Factor Assessment and a Ten-Year Experience of DDH Screening in a Well-Child Population. Biomed Res Int 2019;2019:7213681.

16. Adams AJ, Johnson MA, Ryan KA, et al. Safe Transportation in-Spica Following Surgical Treatment of Infantile DDH: Solutions and Threats. J Pediatr Orthop 2019;39:e488-e493.

17. Vafaee AR, Baghdadi T, Baghdadi A, et al. DDH Epidemiology Revisited: Do We Need New Strategies? Arch Bone Jt Surg 2017;5:440-2.

18. Kothari A, Grammatopoulos G, Hopewell S, et al. How Does Bony Surgery Affect Results of Anterior Open Reduction in Walking-age Children With Developmental Hip Dysplasia? Clin Orthop Relat Res 2016;474:1199-208.

19. Vafaeian B, Zonoobi D, Mabee $M$, et al. Finite element analysis of mechanical behavior of human dysplastic hip joints: a systematic review. Osteoarthritis Cartilage 2017;25:438-47.

20. Memeo A, Panuccio E, D'Amato RD, et al. Retrospective, 
multicenter evaluation of complications in the treatment of diaphyseal femur fractures in pediatric patients. Injury 2019 Aug;50 Suppl 4:S60-3.

21. Klein C, Fontanarosa A, Khouri N, et al. Anterior and lateral overcoverage after triple pelvic osteotomy in childhood for developmental dislocation of the hip with acetabular dysplasia: Frequency, features, and mediumterm clinical impact. Orthop Traumatol Surg Res 2018;104:383-7.

22. Sonoda K, Motomura G, Ikemura S, et al. Effects of intertrochanteric osteotomy plane and preoperative femoral anteversion on the postoperative morphology of the proximal femur in transtrochanteric anterior rotational osteotomy: 3D CT-based simulation study. Orthop Traumatol Surg Res 2017;103:1005-10.

Cite this article as: Qiu S, Lin H, Xu M, Liu C, Wang H, Cao Q, Liu J. Effect of acetabular morphological parameters applied in proximal femoral varus osteotomy on the treatment of developmental dysplasia of the hip in children. Transl Pediatr 2021;10(5):1361-1368. doi: 10.21037/tp-21-200
23. Umer M, Nawaz H, Kasi PM, et al. Outcome of triple procedure in older children with developmental dysplasia of hip (DDH). J Pak Med Assoc 2007;57:591-5.

24. Pascual-Garrido C, Guilak F, Rai MF, et al. Canine hip dysplasia: A natural animal model for human developmental dysplasia of the hip. J Orthop Res 2018;36:1807-17.

25. Zhou W, Sankar WN, Zhang F, et al. Evolution of concentricity after closed reduction in developmental dysplasia of the hip. Bone Joint J 2020;102-B:618-26.

26. Gaffney BMM, Hillen TJ, Nepple JJ, et al. Statistical shape modeling of femur shape variability in female patients with hip dysplasia. J Orthop Res 2019;37:665-73.

(English Language Editor: J. Jones) 